\title{
Zur Konstitution des Gegenstandsbereichs der Geographie bei Hans Carol
}

Diese Arbeit hat drei Teile. Zunächst werde ich einige grundsätzliche Bemerkungen zur Konstitution von wissenschaftlichen Gegenstandsbereichen machen, dann die allgemeinen Resultate mit dem Ansatz von CAROL vergleichen und schließlich auf einige Kritik eingehen, die am CARoLschen Ansatz geübt worden ist.

\section{I.}

Ich beginne die Diskussion darüber, wie wissenschaftliche Gegenstandsbereiche konstituiert werden, mit einer Klärung des Wissenschaftsbegriffs der empirischen Wissenschaften. Allerdings strebe ich keine so weitgehende Klärung an, daß an ihrem Ende eine Definition von Wissenschaft steht - ich werde nur so weit gehen, wie das für unsere Zwecke notwendig ist. Was meinen wir eigentlich mit Wissenschaft? Welche Forderungen muß etwas, das den Namen Wissenschaft zu Recht trägt, eigentlich erfüllen? Eine Antwort, die sehr vorläufig ist, mit der wir aber einsetzen können, lautet: Wissenschaft ist systematisches Wissen. Vorläufig ist diese Antwort deshalb, weil auch die Begriffe Wissen und systematisch klärungsbedürftig sind.

1. Was ist nun Wissen oder Erkenntnis? Zunächst sieht man sofort, daß Wissen immer Wissen von etwas ist, Wissen sich also immer auf irgendwelche Objekte bezieht. Objekt oder Gegenstand ist hier in einem sehr weiten Sinn zu nehmen: es muß keineswegs ein materieller Gegenstand sein, der Objekt des Wissens ist. So hat beispielsweise die Primzahlforschung die Primzahlen als ihre Objekte, und wir haben im Moment so etwas wie die Idee der Wissenschaft als unser Objekt. Wissen hat weiterhin (zumindest potentiell) ein Subjekt, das ist hier schlicht gesprochen einfach der Mensch, der etwas weiß. Der Ausdruck «Subjekt» ist ein traditioneller Ausdruck der Erkenntnistheorie, auf dessen Geschichte und genauere Bedeutung ich hier nicht eingehen kann. Unser erstes Resultat lautet also: Wissen ist eine Relation zwischen einem Subjekt und einem Objekt.

Es gibt nun aber viele Relationen von einem Subjekt auf ein Objekt, z.B. vorstellen, begehren, schön finden, verachten usw., und wir müssen uns daher fragen, was das Spezifische der Wissensrelation ist, was die Wissensrelation von den anderen Relationen von einem Subjekt auf ein Objekt unterscheidet. Das kann man sich klarmachen, indem man eine Relation aufsucht, die der Wissensrelation entgegengesetzt ist. Das ist das bloße Glauben, das bloß subjektive Meinen. Schärfer: Das bloß subjektive Meinen ist dem objektiven Wissen entgegengesetzt. In beiden Relationen ist eines der Relate der Relation besonders angesprochen, nämlich das, auf dem gewissermaßen der Akzent liegt: beim bloßen Meinen auf dem Subjekt und beim Wissen auf dem Objekt. Die Relation Wissen soll also wesentlich durch das Objekt des Wissens bestimmt sein, nicht so sehr durch die Beiträge des Subjekts, wie beim bloßen Meinen. Im Wissen soll das Objekt eben so dargestellt werden, wie es an sich ist, unabhängig von mir. Nicht so, wie es mir zufällig erscheint, wenn ich nicht genau hinsehe oder voller verzerrender Vorurteile bin, oder übermüdet, oder betrunken.

Daraus läßt sich sehen, daß Wissen ausweisungsbedürftig ist: wenn jemand $\mathrm{W}$ issen beansprucht, dann darf man immer fragen: Woher weißt Du das? Und der Wissensanspruch muß ausgewiesen werden mit Rekurs auf das Objekt, nicht mit Rekurs auf das Subjekt allein, wie das bei der Antwort «Das meine ich einfach so" der Fall wäre. Der Grund für die Ausweisungsbedürftigkeit des Wissens ist, daß die Wissensrelation nicht immer gelingt: es gibt den Irrtum, bei dem man das Objekt irgendwie verfehlt. Der Wissensausweis muß zeigen, daß die Relation auf das Objekt nicht durch das Subjekt verfälscht wird.

Noch eine trivial scheinende Bemerkung. In der Wissensrelation wird als selbstverständlich vorausgesetzt, daß es das Objekt gibt. Etwas, was es nicht gibt, kann nicht inhaltlich Gegenstand unseres Wissens sein. Vom runden Quadrat kann man nur wissen, daß es nicht existiert. Traditionell gesprochen: im Wissen wird das Dasein des Objekts vorausgesetzt.

Soviel zum Begriff Wissen. Der zweite klärungsbedürftige Begriff war systematisch.

2. In der Aussage, Wissenschaft sei systematisches Wissen, wird mit «systematisch» mindestens dreierlei gemeint.

Paul Hoyningen-Huene

Philosophisches Seminar der Universität Zürich

Rämistr. 71, 8006 Zürich 
a) Der Wissensausweis, von dem ich gerade vorhin sprach, hat in der Wissenschaft nach mehr oder weniger stabilen und mehr oder weniger bewußten Regeln zu erfolgen, d.h. methodisch, oder eben: systematisch. Es gibt also Verfahren des Wissensausweises, die man einzuhalten hat. Beispielsweise gilt in der Naturwissenschaft die Grundregel, daß der Wissensausweis sich in letzter Instanz auf das Experiment zu berufen hat. Worauf beruht die Geltung solcher Regeln des Wissensausweises? Anders gefragt, wie werden die wissenschaftlichen Methoden begründet? Darauf komme ich später zu sprechen.

b) Bei der Aussage, Wissenschaft sei systematisches Wissen, ist bei "systematisch» eine zweite Bedeutungskomponente beteiligt: wissenschaftliches Wissen ist in seiner Gesamtheit nicht unstrukturiert. Wissenschaftliches Wissen ist kein beliebiges Aggregat von Einzelwissen, sondern gemäß Gegenstandsbereichen (oder Objektbereichen) gegliedert: eine Einzelwissenschaft bezieht sich immer auf eine Sorte von Objekten. Wie wird nun ein Objektbereich einer Wissenschaft ausgegrenzt aus der Totalität aller möglichen Objekte? Wie erfolgt die Zusammenfassung von Objekten zu einem Objektbereich, so daß ich später sagen kann, das Wissen der Wissenschaften insgesamt sei systematisch gegliedert, d. h. mit oder nach System gegliedert? Es sind zunächst zwei prinzipiell entgegengesetzte Möglichkeiten denkbar, woher die Gesichtspunkte kommen können, die für die Ausgrenzung eines Objektbereichs bestimmend sind:

- von den Objekten selbst her, z. B. die tatsächliche Gleichheit oder Ähnlichkeit der Objekte,

- nicht von den Objekten des Wissens her, sondern den Subjekten, z.B. Relevanzgesichtspunkte oder Konventionen.

Beide Arten von Gesichtspunkten spielen bei der Ausgrenzung von wissenschaftlichen Objektbereichen eine Rolle.

Zunächst einige Beispiele. Daß es eine eigene Wissenschaft von den Zahlen gibt, die Arithmetik, daß es eine eigene Wissenschaft von den Tieren gibt, die Zoologie, daß es eine eigene Wissenschaft von den romanischen Sprachen gibt, die Romanistik, scheint bloß daran zu liegen, daß die jeweiligen Objekte der Wissenschaft untereinander starke Ähnlichkeiten aufweisen, und gegen die Objekte anderer Wissenschaften starke Unähnlichkeiten. Von daher lassen sie sich leicht ausgrenzen. $\mathrm{Da} ß$ es aber keine Wissenschaft von den zwei Meter langen Dingen gibt (R.SPAemanN), zeigt, daß wir die objektiv bestehenden Ähnlichkeiten von Objekten auch interessant oder relevant finden müssen, damit wir sie in einen Objektbereich einer Wissenschaft zusammenfassen. Es spielen bei der Ausgrenzung eines Objektbereichs einer Wissenschaft also objektbestimmte und subjektbestimmte Momente eine Rolle. Das sieht man deutlich daran, wie ein materielles Ding Objekt verschiedener Wissenschaften sein kann, also verschie- denen Objektbereichen zugerechnet werden kann. Ein Stein mit eingeritzten Schriftzeichen zum Beispiel kann Objekt der Physik sein, etwa wenn man nach seinem Gewicht oder seiner Elastizität fragt. Er kann Objekt der Chemie sein, wenn seine chemische Zusammensetzung interessiert. Ebenso kann er unter entsprechenden Fragestellungen Objekt der Kristallographie, der Geologie, der Archäologie, der vergleichenden Sprachwissenschaften und sogar der Kriminologie sein, wenn jemand mit diesem Stein eine Schaufensterscheibe eingeschlagen hat.

Die subjektbestimmten und die objektbestimmten Momente greifen bei der Konstitution von wissenschaftlichen Gegenstandsbereichen also ineinander: ich habe einen Relevanzgesichtspunkt (subjektiv), und erst dann kann sich ein Objekt als einem Gegenstandsbereich zugehörig erweisen (objektiv). Ich muß zunächst anmelden, was mich am Objekt interessiert, und erst dann kann die Antwort vom Objekt her erfolgen. Noch ein vielleicht paradox scheinendes Beispiel: auch die Tierganzheit, die den Objektbereich der Zoologie bestimmt, ist nicht bloß vom Objekt her vorgegeben. Das sieht man an der Möglichkeit anderer ebenso objektiver Beschreibungsmöglichkeiten eines Ökosystems, die von anderen Interessen geleitet sind. So könnte man ein Ökosystem rein physikalisch etwa durch die Kreisläufe der Sauerstoff-, Kohlenstoff- und Stickstoffatome beschreiben, und der Aufenthalt der Atome in den Tieren ist nicht besonders ausgezeichnet. Erst das Interesse für das Lebendige und weitere Relevanzgesichtspunkte lassen die einzelnen Tierganzheiten identifizieren.

Die Konstitution eines wissenschaftlichen Gegenstandsbereichs ist also vom Subjekt her und den Objekten her bestimmt. Das letztere heißt aber nichts anderes, als daß ich über die Objekte einer Wissenschaft schon etwas wissen muß, um sie überhaupt in einen Objektbereich zusammenfassen zu können. Dieses Wissen geht über das Wissen um das bloße Dasein der Objekte hinaus: daß diese Objekte nämlich unter einem gewissen Blickwinkel gemeinsame Eigenschaften haben. Traditionell gesprochen: ich benötige Wissen über das Sosein der Objekte. In diesem Sinne beginnt Wissenschaft nicht voraussetzungslos: zur Vereinigung von Objekten in einen Objektbereich wird bereits Wissen um ihr Dasein sowie manches Wissen um ihr Sosein vorausgesetzt. Auch das mag merkwürdig klingen, wird aber an Beispielen sofort klar:

- Die Molekularbiologie konnte erst entstehen, nachdem man wußte, daß es biologisch relevante Makromoleküle gibt.

- Die Tiefenpsychologie hat zur Voraussetzung, daß es das Unbewußte gibt, und $d a ß$ es mit dem Bewußtsein wechselwirkt.

- Die Astronomie setzt, wenn sie etwa die gesetzmäBige Bewegung der Himmelskörper erforschen will, die Existenz von Himmelskörpern sowie Gesetze ihrer Bewegung voraus. 
In allen Fällen wird also Wissen über Dasein und Sosein der Objekte vorausgesetzt.

Man kann nun auch sehen, woher das Wissen stammen kann, das man bei der Konstitution eines wissenschaftlichen Gegenstandsbereichs voraussetzen muß. Es stammt aus Vorgänger- oder Nachbarwissenschaften oder aus beliebigen anderen Bereichen menschlicher Praxis, in denen Wissen erzeugt wird, also z. B. alltäglicher (bzw. allnächtlicher) Himmelsbeobachtung, handwerklicher oder medizinischer Praxis.

Dieses Wissen um Dasein und Sosein der Objekte spielt auch bei der Begründung der fundamentalen Methoden einer Wissenschaft eine entscheidende Rolle. Beispielsweise begründet sich die Forderung nach Reproduzierbarkeit von Experimenten in der Physik oder Testergebnissen in der Psychologie in der relativen Konstanz der relevanten Objekteigenschaften. Die Tatsache, daß solches Wissen beim In-GangSetzen einer Wissenschaft vorausgesetzt werden muß, schließt dabei nicht aus, daß dieses Wissen im Verlauf der Wissenschaft präzisiert und sogar revidiert werden kann, wie man am Beispiel der großen Umwälzungen in der Physik dieses Jahrhunderts leicht zeigen könnte.

c) Schließlich ist eine dritte Bedeutungskomponente von systematisch zu nennen, die in der Aussage, Wissenschaft sei systematisches Wissen, beteiligt ist. Gemeint ist damit, daß Wissenschaft ein Ideal von Vollständigkeit des Wissens über den jeweiligen Objektbereich hat. Man möchte «alles» über den Objektbereich wissen. Das ist mit einigen begrifflichen Schwierigkeiten verbunden, auf die ich hier aber nicht eingehen kann.

Ich fasse die bisherigen Ergebnisse zusammen:

Wissenschaft wurde bestimmt als systematisches Wissen. Wissen ist eine objektbestimmte SubjektObjekt-Relation. Systematisch heißt hier, daß das Wissen methodisch überprüft wird, daß es in Wissensbereiche gegliedert ist, die zu subjekt- und objektbestimmten Gegenstandsbereichen korrespondieren, und daß Wissenschaft ein Vollständigkeitsideal hat.

Aus der bisherigen Erörterung lassen sich nun vier Forderungen ableiten, die man an eine durchsichtige Grundlegung einer Wissenschaft im Sinne der Konstitution ihres Gegenstandsbereichs stellen kann.

1. Es ist das Wissen auszuweisen, das das Dasein der Objekte behauptet.

2. Es ist der subjektive Blickwinkel anzugeben, unter dem diese Objekte in einen Gegenstandsbereich zusammengeschlossen werden sollen.

3. Es sind diejenigen Eigenschaften der Objekte anzugeben und auszuweisen, die den Objektbereich dann tatsächlich konstituieren (Sosein der Objekte).

4. Es sind diejenigen Eigenschaften der Objekte zu nennen und auszuweisen, die zur Begründung der fundamentalen Methoden der jeweiligen Wissenschaft benötigt werden.
Diese Forderungen können wegen der philosophischen Probleme, die in ihnen impliziert sind, nicht vollständig erfüllt werden. Wegen der Verschränktheit dieser Forderungen können sie auch nicht säuberlich getrennt voneinander behandelt werden. Aber sie können als Leitfaden für die Grundlegung einer Wissenschaft dienen, und sie können einen Qualitätsvergleich verschiedener Grundlegungsversuche für eine Wissenschaft ermöglichen.

\section{II.}

Mit diesem Leitfaden möchte ich nun die Konstitution des Gegenstandsbereichs der Geographie untersuchen, wie sie H.CAROL 1956 in seiner Arbeit «Zur Diskussion um Landschaft und Geographie» vorgelegt hat (Geogr. Helv. 11111 [1956], wiederabgedruckt in W.Storkebaum [Hg.]: Zum Gegenstand und zur Methode der Geographie [Wissenschaftliche Buchgesellschaft, Darmstadt, 2/1975]. Ich gebe im folgenden zuerst die Seitenzahl des Originals, dann die des Abdrucks an).

Carols Arbeit ist in drei große Abschnitte gegliedert (S. 111/S.475). Der erste Abschnitt über das Objekt geographischer Forschung behandelt u.a. unsere Forderungen 1., 2. und 3., der zweite Abschnitt geht u. a. auf die 4. Forderung ein, und der dritte Abschnitt setzt sich mit neuerer Literatur zum Thema auseinander.

Der eigentliche Beginn der Arbeit ist in Abschnitt I.2 (113/478ff.), wo das Objekt der Geographie behandelt wird. Dort sagt CAROL (113/478): "Tatsächlich, die Erdhülle ist das Objekt geographischer Forschungen, ...» Die Frage nach dem Dasein der Erdhülle wird gar nicht gestellt, denn sie ist wohl im Kontext wissenschaftlichen Fragens nicht ernsthaft zu stellen. Auch das hier schon vorausgesetzte Sosein der Erdhülle, nämlich ihre Kugelgestalt, wird nicht in Frage gestellt, obwohl auch sie alles andere als selbstverständlich ist, wie die Geschichte der Wissenschaft lehrt. Dennoch wird niemand in einer wissenschaftlichen Abhandlung aus dem Jahre 1956 eine Auflistung der Argumente erwarten, die die Hypothese von der Kugelgestalt der Erde plausibel machen. Direkt anschließend an die zitierte Stelle bringt $C_{A R O L}$ weitere Bestimmungen des Objekts, also Aussagen über sein Sosein (113/478): «Fünf wesentliche Bereiche, Sphären, sind an ihrem Aufbau beteiligt: Litho-, Hydro-, Atmo-, Bio- und Anthroposphäre, ...» Auch diese Aussage über das Objekt ist nicht ausgewiesen. Das liegt daran, daß ihr uns allen bekannte und sicher handhabbare Unterscheidungen zugrundeliegen: zuerst die Unterscheidung von unbelebt und belebt, dann die Unterteilung des Unbelebten in fest, flüssig und gasförmig und schließlich die Aufteilung des Belebten in Menschliches und Nicht-Menschliches. Damit sind die fünf Sphären gewonnen.

Die nächste Bestimmung des Objekts ist relativ zur vorausgesetzten Kugelgestalt der Erde und betrifft 
ebenfalls als trivial und selbstverständlich angesehenes Wissen (113/478): «Die Erdhülle ist an jedem Orte aus der Gesteinskruste und der Lufthülle, und dazu in nicht zusammenhängender Form - auch aus der Wasserhülle aufgebaut. Die erstgenannten anorganischen Hüllen umschließen die Erde kontinuierlich. Das organische Leben, wie auch das Reich des Menschen, fehlen in gewissen Teilen der Erdhülle.» Die jetzt folgende Bestimmung weist die Möglichkeit einer Grenzbestimmung innerhalb der Erdhülle rein vom Objekt her zurück (113/479): «Die einzelnen Sphären differenzieren sich ständig, aber nirgends entsteht eine Grenze, die durch alle Sphären reichte, deren trennende Wirkung eine eindeutige Teilung der Erdhülle zustandebrächte. Diese ist vielmehr ein reichgegliedertes Kontinuum, dem insgesamt ein ganzheitlicher Charakter zukommt.» Weiter unten findet sich eine ebenso deutliche Formulierung (113/479): "Wenn es keine von der Natur gegebene, alle Sphären der Erdhülle umfassende Gliederung gibt, kann es auch keine „Landschaften” im Sinne von "Raumorganismen" als isolierte Forschungsobjekte der Geographie geben.» Das Argument ist strukturgleich mit dem Hauptargument von denjenigen Historikern, die Epocheneinschnitte als nicht objektiv, sondern als konventionell einschätzen: wenn es zu allen Diskontinuitäten überbrückende Kontinuitäten gibt, wenn es also Diskontinuität nur in der Kontinuität gibt, dann lassen sich Einschnitte eben nicht vom Objekt allein her gewinnen, sondern nur, indem man den Diskontinuitäten vom Subjekt her größeres Gewicht verleiht. Die Notwendigkeit eines subjektiven Beitrags wird von CAROL deutlich gesehen (114/479): «Da unser Interesse aber nicht der Erdhülle als Ganzes gilt, sondern weit häufiger kleineren Teilbereichen (Kontinenten, Ländern, Teilen von Ländern), so ist ihre Unterteilung aus praktischen Gründen nötig. Das Kontinuum Geosphäre kann nach irgendwelchen Kriterien zerlegt werden...» Diese Stelle ist nun etwas mißverständlich, da mit der Rede von «irgendwelchen Kriterien» der Eindruck entstehen kann, als würde hier der Willkür Tür und Tor geöffnet, und der Gegenstand völlig bedeutungslos würde, damit auch die Objektivitätsforderung aufgegeben sei. Tatsächlich kann zunächst vom Objekt her über mögliche Relevanzgesichtspunkte nichts gesagt werden. Eine andere Stelle bringt Klärung (126/503): «Maßgebend für die Abgrenzung [der zu untersuchenden Landschaft, P.H.] ist der Zweck der Untersuchung. Interessiert mich die Landschaft der Zürichseetalung, so wird die Untersuchungsgrenze zweckmäßigerweise auf die benachbarten Wasserscheiden gelegt,... Interessiert mich die Landschaft der Gemeinde Osterfingen oder die Landschaft der Schweiz, so ist das Untersuchungsgebiet politischadministrativ begrenzt,...» Hier wird folgendes deutlich: wenn der Zweck einmal festliegt, dann ist die Willkür vom Objekt her aufgehoben. Die Formulierungen «zweckmäßigerweise» und «ist... begrenzt» im gerade zitierten Passus legen diese Deutung nahe. Der Sache nach scheint mir diese Deutung zwingend, da die Situation strukturgleich mit dem früher angeführten Beispiel des mit Schriftzeichen bedeckten Steines ist. Welcher Aspekt des Steines mich interessiert, ist tatsächlich meiner Willkür überlassen. Habe ich mich einmal entschieden, so ist der Stein als Objekt einer Einzelwissenschaft fixiert. Jetzt habe ich keinerlei Willkür mehr: das Objekt schreibt mir vor, was die relevanten Eigenschaften sind, diese bestimmen in letzter Instanz die Untersuchungsmethoden, und die Ergebnisse der Untersuchung sind - jedenfalls solange gewisse quantenmechanische Effekte keine Rolle spielen - gänzlich meiner Willkür entzogen und allein vom Objekt her bestimmt.

Damit ist man beim Geomer-Begriff angelangt. Die weiteren Bestimmungen dienen der Fixierung der spezifisch geographischen Aufgabe (115/483): «Wären die Elementarsphären unverbunden überschichtet, so würde die elementarwissenschaftliche Forschung zur Erklärung der Geosphäre genügen - eine Geographie wäre überflüssig. Tatsächlich kommen aber alle Grade von Verbindungen zwischen den Sphären, extensive bis sehr intensive, vor.» Ausgewiesen wird diese Behauptung im Anschluß mit unbezweifelbaren Beispielen. Das ist vollkommen ausreichend, da etwa eine systematische Beschreibung, eine Klassifikation oder sogar Erklärung der Korrelationen gerade Forschungsziel ist und bei der Konstitution des wissenschaftlichen Gegenstandsbereichs nicht geleistet zu werden braucht. Nachdem nun das Objekt der Geographie fixiert ist, kommt CAROL im zweiten Abschnitt zunächst zum Wissenschaftsbegriff. Die «wissenschaftlich-rationale Betrachtung...sucht eine dem Objekt gerecht werdende Schau» (117/487). Das ist dasselbe, was ich mit anderen Worten beim Wissensbegriff entwickelt habe. Das für uns in diesem Zusammenhang etwas altmodisch klingende Wort «Schau» drückt dabei gerade die Passivität des Subjektes aus: es darf in die Wissensrelation nicht störend eingreifen. (Wieweit man, um menschliche Erkenntnis durchsichtig zu machen, eine sehr spezifische aktive Beteiligung des Erkenntnissubjekts ansetzen muß, ist ein Zentralproblem der Erkenntnistheorie. Das muß uns hier aber nicht weiter beschäftigen: Wissenschaft kann nur in Gang kommen, wenn man nicht alle philosophischen Probleme vorgängig zu behandeln versucht - sonst «ersäuft» man nämlich in der Philosophie.)

Die Gliederung der Geographie in Subdisziplinen ist durch die Gliederung der Erdhülle in die verschiedenen Sphären vorgegeben (118ff./488ff.). Die einzelnen Subdisziplinen haben sich methodisch der speziellen Natur ihres Gegenstands anzupassen (119/489) - dies entspricht dem früher Gesagten. CAROL stellt die systematisch zu entwickelnden Subdisziplinen der Geographie z. T. als Desiderata dar - ich gehe daher auf die Details nicht weiter ein.

Ich fasse das Resultat zusammen. CAROL liefert eine 
durchsichtige, die grundlegenden Fragen adäquat behandelnde Darstellung der Konstitution des Gegenstandsbereichs der Geographie. Der subjektive Anteil bei der Ausgrenzung der Geomere erscheint m.E. zu Unrecht im Widerspruch zum Objektivitätsideal der Wissenschaft (selbst CAROL spricht von «der arationalen Komponente der Landschaft» [117/487]). Er ist bei jeglicher wissenschaftlicher Betrachtung beteiligt, weil wir Objekte nicht auf einmal in der Totalität ihrer Eigenschaften thematisieren können - wir sind stets auf Auswahlgesichtspunkte angewiesen. Am Beispiel des Steines mit den Schriftzeichen ließ sich ablesen, daß unsere Interessen, also der subjektive Anteil, nur in die Auswahl der jeweiligen Disziplin eingeht; er bleibt deshalb in der Disziplin unbemerkt. Bei der Geographie wird er innerhalb der Disziplin sichtbar und deshalb bewußt. Man kann sogar noch weiter gehen und feststellen, daß aller Wissenschaft eine "arationale Komponente» im Carolschen Sinn zugrunde liegt: wir können uns als Menschen auf vielerlei Weise zu den Gegenständen dieser Welt verhalten, die neuzeitlich-wissenschaftliche Betrachtung ist nur eine unter vielen Möglichkeiten. Erst wenn wir uns Zwecke setzen, für die Naturwissenschaft etwa den Erwerb von instrumentell kontrollierbarem Wissen über Naturzusammenhänge, wird auch von den jeweiligen Objekten her das weitere Vorgehen bestimmt.

$\mathrm{Zu}$ bemerken ist noch, daß die CARolsche Analyse weder ein Paradigma im Sinne Thомas Kunss noch eine Methodologie oder ein Forschungsprogramm im Sinne ImRe LaKatos' darstellt. CAROL behandelt die logisch frühere und von der heutigen Wissenschaftstheorie weitgehend vernachlässigte Frage der Konstitution eines wissenschaftlichen Gegenstandsbereichs.

\section{III.}

Die jetzt folgende Diskussion der Kritik an CAROL ist fragmentarisch. Ich behandle vier verschiedene Kritikpunkte.

1. E.Száva-Kováts behauptet in seinem Artikel «Das Problem der geographischen Landschaft) (Geogr. Helv. 1538 [1960]), «daß der geographische Landschaftsbegriff... eine erkenntnistheoretisch fehlerhafte Konstruktion darstellt». Denn «er hat keinen konkreten Realitätsgehalt und ihm entspricht auch kein objektiv konkretisierbarer Gegenstand. Die geographische Landschaft ist als Erscheinung eine von unserem Bewußtsein abhängige subjektive Wirklichkeit, ..." (S. 47). Warum ich die subjektive Komponente beim Landschaftsbegriff für keineswegs fehlerhaft, sondern für völlig legitim halte, habe ich vorhin dargelegt.

2. G. HARD nimmt in seinem Buch «Die Geographie. Eine wissenschaftstheoretische Einführung» (de Gruyter, Berlin, 1973) eine Bemerkung von D. BARTELS auf, wonach die heutige «Malaise der Geogra- phie», die «eine (Folge-)Erscheinung mangelnder Integration einer Forschungsdisziplin in das bestehende Sozialsystem" sei, mit dem Festhalten am landschaftsgeographischen Ansatz zusammenhänge (S. 159). Wie immer es mit dieser «Malaise» steht - die "mangelnde Integration in das Sozialsystem» sollte kein Anlaß sein, einen gut begründeten Ansatz einer Wissenschaft aufzugeben. Weder Ludwig BoltzMANN, einer der Begründer der statistischen Mechanik, noch Gotтlob Frege, der Hauptbegründer der modernen mathematischen Logik, waren «in das Sozialsystem integriert»: sie erhielten ihr Leben lang fast keine Anerkennung - heute werden sie als geniale Wissenschaftler gefeiert. Die guten Gründe sollten in der Wissenschaft Vorrang haben vor der Anerkennung durch die Massen.

3. G. HARD bemerkt an anderer Stelle des genannten Buches (S. 166), daß dem "ganzheitlichen Landschaftsbegriff» der offenkundige Sachverhalt widerspricht, «daß sich zahlreiche Merkmale zahlreicher landschaftlicher Phänomene...völlig oder weitestgehend unabhängig voneinander entwickeln». H. WANNER fügt dem bei: «Die Anthroposphäre hat sich im 20. Jahrhundert durch die technische und gesellschaftliche Entwicklung aus der Integration mit der Geosphäre gelöst. Deshalb erklärt CARoLs Ansatz mit den Korrelationen zwischen den Sphären die modernen Phänomene in der Anthroposphäre schlecht» (persönl. Mitteilung 1980). Dem genannten Faktum ist vermutlich ohne Vorbehalt zuzustimmen. Das widerspricht dem Carolschen Ansatz überhaupt nicht - im Gegenteil: der Carolsche Ansatz könnte den Boden legen für eine außerordentlich interessante detaillierte Untersuchung der Emanzipation der Anthroposphäre von den anderen Sphären in den letzten Jahrhunderten. Ein fruchtbares Forschungsfeld für die Kooperation zwischen Geographen, Historikern, ... Gäbe es übrigens die genannte relative Unabhängigkeit der Sphären nicht, so gäbe es außer einer Geographie im CAROLschen Sinne überhaupt keine anderen Wissenschaften. Bei jedem in einer Sphäre beheimateten Phänomen müßten die Einflüsse aus anderen Sphären wesentlich berücksichtigt werden - und genau das wäre CARoLsche Geographie.

4. U.EIsel stellt in seiner Arbeit «Die Entwicklung der Anthropogeographie von einer „Raumwissenschaft" zur Gesellschaftswissenschaft» (Urbs et Regio 17 [1980]) fest, daß das Wort «Landschaft» in der "nationalistischen und faschistischen Ideologie» eine "gesellschaftliche Funktion» besessen habe. Die Konnotationen von Landschaft: Heimat und Vaterland hätten eine Rolle gespielt (S. 135f.). Wie immer es darum steht: weder Konnotationen von Worten noch Mißbrauch von Begriffen in der Politik sind strikte Argumente beim Aufbau einer Wissenschaft. Die Physiker wären schlecht beraten, den Trägheitsbegriff aufzugeben, nur weil die Physikdidaktiker notorisch klagen, die Schüler würden am Anfang immer 
«Faulheit» mit ihm assoziieren (das Beispiel ist nicht frei erfunden). Die Biologen und Anthropologen wären schlecht beraten, den Rassebegriff aufzugeben, weil besonders zwischen 1933 und 1945 wirklich katastrophaler Mißbrauch damit getrieben wurde.

Zusammenfassend: Es scheint mir, daß der Ansatz von $\mathrm{C}_{\mathrm{AROL}}$ ein durchaus tragfähiges Fundament für eine eigenständige Wissenschaft Geographie abgibt allerdings nur unter einer einschränkenden Bedin- gung: daß man der Geographie nicht mehr zumutet, als man es anderen Wissenschaften gegenüber tut: beispielsweise einer so ehrwürdigen Wissenschaft wie der Physik.

\section{Danksagung}

Ich danke $H$. WANNER für Literaturangaben und Diskussionsmöglichkeit und P.Schulthess für seine kritische Lektüre dieses Manuskripts.

\section{Diskussion zum Vortrag von P. Hoyningen}

\author{
Teilnehmerliste \\ PH Dr. PAUL HOYNINGEN, Physiker und Wissenschafts- \\ theoretiker, Zürich (Referent) \\ KI Dr. KLAUS ITTEN, Geograph, Zürich (Diskussionsleiter) \\ HB Dr. HANS R. BRUNNER, Geograph und Soziologe, Zürich \\ PB PETER BÜNZLI, Geographiestudent, Zürich \\ EE Prof. Dr. EMIL EGLI, Geograph, Zürich \\ TK THEO KELLER, Geograph, Zürich \\ RN RUEDI NÄGELI, Geograph, Bern \\ ER ERICH RENNER, Geograph, Zürich \\ CS CHRISTIAN SCHMID, Geographiestudent, Zürich \\ TS THOMAS SCHWEIZER, Geographiestudent, Zürich \\ HW HERBERT WANNER, Geograph, Zürich \\ OW Prof.Dr. OTTO WERNLI, Geograph, Zürich
}

$\mathrm{KI}$ : Wir haben beim letzten Vortrag von Dr.H.R.BRUNNER einiges über die Zürcher Landschaftsschulen erfahren. Bei diesem Referat geht es nun um einen Vertreter der jüngeren Zürcher Landschaftsschule, um HaNS CaROL. Bei dieser Gelegenheit möchte ich noch Herrn Prof. O. WERNLI vorstellen, er war ein Mitstreiter von HANS CaROL und hat in seiner Dissertation zur Diskussion um den Landschaftsbegriff beigetragen. Ich möchte die Diskussion mit einer Frage eröffnen: Darf man das Aufsteigen von feuchten Luftmassen über ein Gebirge und das Ausregnen, ich bezeichne dies als etwas Triviales, heranziehen als Beispiel für eine Korrelation?

PH: Da möchte ich auf zwei Ebenen antworten. Einerseits wird die Existenz von Korrelationen natürlich auch mit trivialen Beispielen bewiesen. Anderseits ist es forschungspsychologisch natürlich besser, wenn man interessante Korrelationen zeigt. $\mathrm{Da}$ der Kohlenstoff eines der wesentlichen Elemente beim Aufbau von Lebewesen ist, hat natürlich nicht die Molekularbiologie ins Laufen gebracht. Dazu hat man interessantere Sachen gebracht, wie die Untersuchung des Hämoglobins. Dort hat man gefunden, wie man aus der Struktur eines Moleküls die biologischen Funktionen ableiten kann, wie es das Molekül schafft, Sauerstoff zu transportieren. Das ist ein interessantes Beispiel. Interessante Korrelationen muß man bringen, damit sich die Leute auch um diese neu konstituierte Wissenschaft kümmern.

PB: Wenn Sie die Prämissen, die Sie für die Wissenschaft genannt haben, aufrechterhalten, dann ist in
Ihrem Wissenschaftsbegriff auch das organisierte Verbrechertum mit enthalten. Die Konsequenz daraus wäre, daß man diese Definition von Wissenschaft etwas enger fassen müßte, z.B. indem man den Begriff des Forschungsprogrammes mit hineinnehmen würde. Wenn man dies tut, so merkt man nämlich, daß die Arbeit von $\mathrm{C}_{\mathrm{AROL}}$ gar nicht in einem Forschungsprogramm liegt. Meine Frage wäre also: Wie können Sie die Forschungslogik von $\mathrm{C}_{\mathrm{AROL}}$ aufzeigen?

PH: Daß die hinreichende Abgrenzung vom organisierten Verbrechertum nicht gemacht ist, finde ich interessant, vielleicht wahr, aber irrelevant. Ich habe jetzt eben einiges gesagt über Wissenschaft, aber längst nicht alles. Ich habe lediglich gesagt, wie man Gegenstandsbereiche konstituiert, ich habe allerdings nicht gesagt, wie man dann, wenn man einen Gegenstandsbereich hat, so etwas wie eine Forschungslogik oder eine Methodologie, wie das auch genannt wird, begründet. In der Tat mag es so sein, daß man diese Ausführungen auch noch auf das organisierte Verbrechertum anwenden kann. Ich glaube, das liegt daran, daß Wissenschaft viel näher am Handwerklichen dran ist, als wir meinen. Wissenschaft ist wirklich aus dem Handwerk entstanden. Und ich habe den Eindruck, $\mathrm{da} ß$ wenn man genau diese Konstitutionsfragen untersucht, dann wird doch Wissen verwendet, ob's nun so handwerkliches Wissen ist oder ob's aus der medizinischen Praxis stammt - es wird halt etwas kontrollierter gemacht als man's sonst tut. So etwas Besonderes ist Wissenschaft gar nicht. Die Tatsache, daß in der theoretischen Physik z. B. der Gleichzeitigkeitsbegriff des Alltags umgestoßen worden ist, schreckt mich dabei gar nicht. Überall startet man mit dem Alltagswissen - in der Wissenschaft, im Handwerk -, und wenn man dann genauer hinsieht, muß man eben einiges davon revidieren.

PB: Die Kritik wäre jetzt natürlich, daß CAROL mit seinem degenerativen Forschungsprogramm in eine Sackgasse hineinführt.

PH: Carol hat hier überhaupt kein Forschungsprogramm vorgelegt. Was er gesagt hat, ist, wie man die 